\title{
Controlled Growth of ZnSe Nanocrystals by Tuning Reactivity and Amount of Zinc Precursor
}

\author{
Lai-Jun Zhang, ${ }^{1}$ Fa-Yun Chen, ${ }^{1}$ Ji-Zao Tong, ${ }^{1}$ Guang-Da Chen, \\ Hai-Jin Huang, ${ }^{1}$ and Xing-Can Shen ${ }^{2}$ \\ ${ }^{1}$ School of Chemistry and Chemical Engineering, Shangrao Normal University, Shangrao 334001, China
${ }^{2}$ Key Laboratory for the Chemistry and Molecular Engineering of Medicinal Resources (Ministry of Education),
School of Chemistry and Chemical Engineering, Guangxi Normal University, Guilin 541004, China \\ Correspondence should be addressed to Lai-Jun Zhang; ljzhang@sru.jx.cn and Xing-Can Shen; xcshen@gxnu.edu.cn
}

Received 31 May 2013; Accepted 17 July 2013

Academic Editor: A. M. S. Silva

Copyright (C) 2013 Lai-Jun Zhang et al. This is an open access article distributed under the Creative Commons Attribution License, which permits unrestricted use, distribution, and reproduction in any medium, provided the original work is properly cited.

\begin{abstract}
Zinc selenide ( $\mathrm{ZnSe}$ ) nanocrystals were synthesized via a phosphine-free route using the highly reactive alkylamine- $\mathrm{H}_{2} \mathrm{Se}$ complex as selenium precursor and zinc precursors with different reactivity. The reactivity of zinc precursor was tuned by using three kinds of zinc carboxylates with different alkyl chain lengths, including zinc acetate, zinc nonanoate, and zinc stearate. The effect of the reactivity and the amount of zinc precursor on nucleation and growth of ZnSe nanocrystals were investigated by ultraviolet-visible absorption and photoluminescence spectra. Result indicates that the growth and optical property of the resulting ZnSe nanocrystals are strongly dependent on the alkyl chain length and the amount of the zinc carboxylates and both shorter alkyl chain length, and more amount of zinc carboxylate will lead to faster growth of $\mathrm{ZnSe}$ nanocrystals. This allows that the controlled growth and excellent optical property of high-quality ZnSe nanocrystals can be achieved by combining the different reactivity and the used amount of zinc precursor, such as by using stoichiometric and reactive $\mathrm{Zn}$ precursor and Se precursor or by using larger amount of more unreactive $\mathrm{Zn}$ precursor relative to the highly reactive alkylamine- $\mathrm{H}_{2} \mathrm{Se}$ complex precursor.
\end{abstract}

\section{Introduction}

Relative to cadmium chalcogenide, zinc chalcogenide is regarded as a "greener" luminescent semiconductor due to the absence of cadmium element, a very toxic heavy metal to human body and natural environment. In recent several years, great development has been made in the synthesis of high-quality zinc selenide nanocrystals (ZnSe NCs) [110]. High-quality ZnSe NCs were usually synthesized at high temperatures by using the stable tri-n-octylphosphine selenide (TOP-Se) or selenium dispersed/dissolved in 1octadecene (ODE-Se) as selenium (Se) precursors to react with various kinds of zinc $(\mathrm{Zn})$ precursors with the proper reactivity, such as the relatively inactive zinc stearate $\left(\mathrm{ZnSt}_{2}\right)$ and zinc oleate [1-4].

Very recently, we made our effort to synthesize highquality metal selenide NCs by appropriately increasing the reactivity of $\mathrm{Se}$ precursor $[11,12]$. The alkylamine- $\mathrm{H}_{2} \mathrm{Se}$ complex, which was generated by adsorbing $\mathrm{H}_{2} \mathrm{Se}$ gas with long-chain alkylamine and used as a more active Se precursor than the traditional TOP-Se and ODE-Se, showed rather high reactivity to zinc nonanoate $\left(\mathrm{Zn}(\mathrm{NA})_{2}\right)$, which results in the formation of magic-sized and even regular $\mathrm{ZnSe} \mathrm{NCs}$ at relatively low temperatures [12]. The nucleation and growth of $\mathrm{ZnSe}$ NCs have been demonstrated to be driven by the reaction thermodynamics and can be tuned successfully by changing the reaction temperature, and four families of $\mathrm{ZnSe}$ magic-sized NCs including F280, F291, F319, and F347 with the first excitonic absorption peaks at 280, 291, 319, and $347 \mathrm{~nm}$, respectively and regular ZnSe NCs were observed in different reaction temperature stages. However, a new problem occurs, that is, whether the $\mathrm{Zn}$ precursor species used will also affect the synthesis and optical properties of the resulting $\mathrm{ZnSe} \mathrm{NCs}$ when using the alkylamine- $\mathrm{H}_{2} \mathrm{Se}$ complex as Se precursor, as can be found in those reaction systems using traditional TOP-Se or ODE-Se precursors [2-4].

In this paper, we investigated the effect of the reactivity and the amount of $\mathrm{Zn}$ precursor on nucleation and growth 
of ZnSe NCs in order to obtain high-quality ZnSe NCs when using the alkylamine- $\mathrm{H}_{2}$ Se complex as an active Se precursor. $\mathrm{Zn}$ precursor species of the same type, that is, zinc carboxylates with different alkyl chain length, including zinc acetate $\left(\mathrm{ZnAc}_{2}\right), \mathrm{Zn}(\mathrm{NA})_{2}$, and $\mathrm{ZnSt}_{2}$, were used for easy comparison and investigation, and the reactivity of $\mathrm{Zn}$ precursor was tuned by changing the alkyl chain length of its carboxylate ligand. In addition, in the previously reported synthesis of $\mathrm{ZnSe} \mathrm{NCs}$ using alkylamine- $\mathrm{H}_{2} \mathrm{Se}$ and $\mathrm{Zn}(\mathrm{NA})_{2}$ as Se and $\mathrm{Zn}$ precursors, respectively, it has been found that $220^{\circ} \mathrm{C}$ may be a key critical temperature because at this temperature, the F347 magic-sized ZnSe NCs will begin to gradually transform into regular NCs [12]. Therefore, the reaction temperature in this work was selected at $220^{\circ} \mathrm{C}$, and at this temperature the effect of different $\mathrm{Zn}$ precursors on the synthesis of ZnSe NCs may be monitored facilely by the ultravioletvisible (UV-vis) absorption and photoluminescence (PL) spectra.

\section{Materials and Methods}

2.1. Chemicals. All commercial reagents were used without any purification. Selenium (Se) powder (99.5\%) and 1octadecene (ODE, 90\%) were purchased from Alfa Aesar. Zinc acetate dehydrate $\left(\mathrm{ZnAc}_{2} \cdot 2 \mathrm{H}_{2} \mathrm{O}, \mathrm{AR}\right)$, octylamine (OA, $99 \%)$, and sodium borohydride $\left(\mathrm{NaBH}_{4}, \mathrm{AR}\right)$ were purchased from Beijing Chemical Plant. Zinc stearate $\left(\mathrm{ZnSt}_{2}, \mathrm{AR}\right), n$ nonanoic acid (NA, 98\%), and oleylamine (OLA, 80-90\%) were purchased from Aladdin Reagent Co., Ltd. Analyticalgrade acetone and cyclohexane were supplied by Shantou Xilong Chemical Co. Ltd.

2.2. Synthesis of $Z n$ and Se Precursors. Prior to synthesis of $\mathrm{ZnSe} \mathrm{NCs}$, Se precursor (alkylamine- $\mathrm{H}_{2}$ Se complex) solution and zinc nonanoate $\left(\mathrm{Zn}(\mathrm{NA})_{2}\right)$ were prepared according to our methods reported previously [12]. Briefly, Se precursor solution was obtained by using a mixture of $\mathrm{OA}(1 \mathrm{~mL})$ and OLA $(1 \mathrm{~mL})$ to absorb $\mathrm{H}_{2} \mathrm{Se}$ gas which was produced by reducing $0.5 \mathrm{mmol}$ of Se powder with $1.5 \mathrm{mmol}$ of $\mathrm{NaBH}_{4}$ under acidic condition. The actual Se amount in the resulting Se precursor solution was estimated to be about $0.4 \mathrm{mmol}$ by weighing the bottle before and after absorbing $\mathrm{H}_{2} \mathrm{Se}$ gas. $\mathrm{Zn}(\mathrm{NA})_{2}$ was synthesized by the reaction of $\mathrm{ZnAc}_{2}$ with NA under reflux conditions.

2.3. Synthesis of ZnSe NCs. Synthesis of ZnSe NCs is detailed as follows. In a $50 \mathrm{~mL}$ three-neck flask, the mixture of $0.4 \mathrm{mmol}$ or $0.6 \mathrm{mmol}$ of $\mathrm{Zn}$ precursor, $2 \mathrm{~mL}$ of ODE, and $1 \mathrm{~mL}$ of OLA were heated at $220^{\circ} \mathrm{C}$ in argon atmosphere. Then, the freshly prepared Se precursor solution was injected rapidly into the hot reaction mixture under vigorous stirring. Finally, $0.1 \mathrm{~mL}$ aliquot of reaction solution was extracted at different time intervals, injected into $0.5 \mathrm{~mL}$ of acetone, and centrifugated at $4000 \mathrm{r} / \mathrm{min}$ for $10 \mathrm{~min}$. The pale-yellow precipitation (ZnSe NCs) was obtained after removing the upper clear liquid, further redissolved by adding a small amount of cyclohexane, and purified repeatedly according to the previous procedure.
2.4. Characterization. Purified ZnSe NCs were dispersed into cyclohexane for ultraviolet-visible (UV-Vis) absorption and photoluminescence (PL) spectra measurements. The absorption spectrum was collected using Varian Cary-100 UV/Vis spectrophotometer in the wavelength range of $250-500 \mathrm{~nm}$ at the scanning speed of $600 \mathrm{~nm} / \mathrm{min}$ with data interval of $1 \mathrm{~nm}$. PL spectrum was collected using Shimadzu RF-5301 PC spectrofluorophotometer using excitation wavelength of $300 \mathrm{~nm}$.

\section{Results and Discussion}

3.1. UV-Vis Absorption Spectrum. Figures 1(a)-1(c) shows the evolution of UV-Vis absorption spectra of ZnSe NCs using $0.4 \mathrm{mmol}$ of $\mathrm{ZnAc}_{2}, \mathrm{Zn}(\mathrm{NA})_{2}$ and $\mathrm{ZnSt}_{2}$ as $\mathrm{Zn}$ precursors, respectively. For these resulting $\mathrm{ZnSe}$ NCs, two sharp and characteristic absorption peaks at about 347 and $328 \mathrm{~nm}$, respectively are generally believed to be the first and second excitonic absorption peaks of F347 magic-sized NCs, and the absorption at longer wavelength originates from the regular $\mathrm{ZnSe} \mathrm{NCs}[7,9,12]$. In the case using $\mathrm{ZnAc}_{2}$ as $\mathrm{Zn}$ precursor, at first these two characteristic absorption peaks at 347 and $328 \mathrm{~nm}$ are observable during the relatively short reaction time and then disappear completely after 20 min, indicating the presence of a small amount of F347 magic-sized $\mathrm{ZnSe} \mathrm{NCs}$ and their transformation into regular $\mathrm{ZnSe}$ NCs. However, when using $\mathrm{Zn}(\mathrm{NA})_{2}$ or $\mathrm{ZnSt}_{2}$ as $\mathrm{Zn}$ precursors, there are always the characteristic peaks of F347 magic-sized NCs within up to 120 min besides the continuous absorptions in the $350-400 \mathrm{~nm}$. In addition, in the case using $\mathrm{ZnSt}_{2}$, not only there remain two characteristic peaks of F347 magic-sized ZnSe NCs, but also the intensity ratio of the characteristic peak to the continuous absorption during 350$400 \mathrm{~nm}$ becomes higher than that in the case using $\mathrm{Zn}(\mathrm{NA})_{2}$ with the same reaction time, indicating that these $\mathrm{ZnSe} N \mathrm{NCs}$ obtained using $\mathrm{ZnSt}_{2}$ as $\mathrm{Zn}$ precursor correspondingly have higher ratio of $\mathrm{F} 347$ magic-sized $\mathrm{ZnSe} \mathrm{NCs}$ to regular $\mathrm{ZnSe}$ NCs in the amount. This result should be related to different alkyl chain lengths of their carboxylate ligands of three kinds of Zn precursors [13]. Generally, the strong and large coordinating ligand will inhibit the growth of semiconductor NCs due to the steric effect. For these three kinds of zinc carboxylates, the order of the alkyl chain lengths of their carboxyl groups is as follows: $\mathrm{ZnAc}_{2}<\mathrm{Zn}(\mathrm{NA})_{2}<\mathrm{ZnSt}_{2}$. Obviously, $\mathrm{ZnAc}_{2}$ and $\mathrm{ZnSt}_{2}$ have minimum and maximum steric hindrance effects, respectively. Therefore, there are the lowest and highest amount ratios of magic-sized to regular $\mathrm{ZnSe} \mathrm{NCs}$ when using $\mathrm{ZnAc}_{2}$ and $\mathrm{ZnSt}_{2}$ as $\mathrm{Zn}$ precursors, respectively. The above results show that the $\mathrm{Zn}$ precursor species have very important effect on the nucleation and growth of ZnSe NCs and the used zinc carboxylate precursor with longer alkyl chain usually results in slower growth of ZnSe NCs.

The nucleation and growth process of $\mathrm{ZnSe} \mathrm{NCs}$ were affected not only by the type of $\mathrm{Zn}$ precursor but also by the amount of these Zn precursors. Figures 1(d)-1(f) show UVVis absorption spectra of ZnSe NCs using $0.6 \mathrm{mmol}$ of three kinds of Zn precursors when fixing other conditions including the amount of Se precursor. As described previously, 


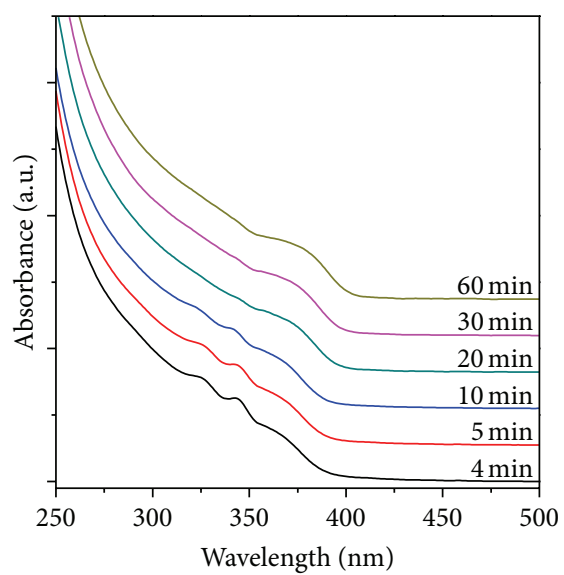

(a)

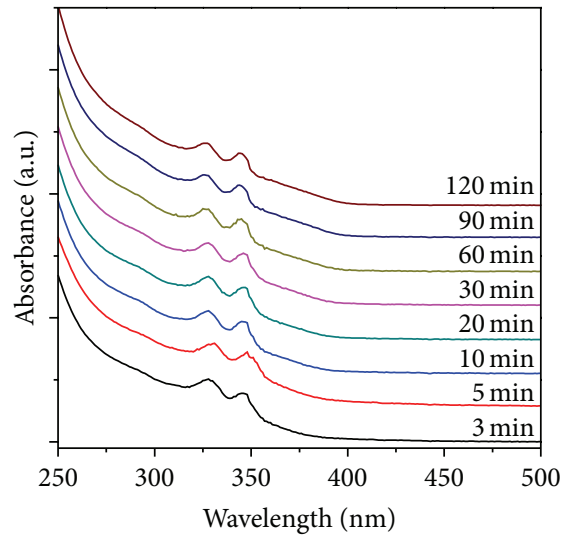

(c)

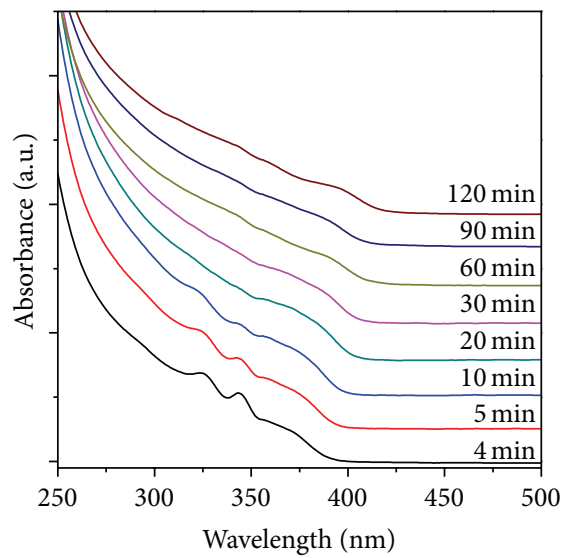

(e)

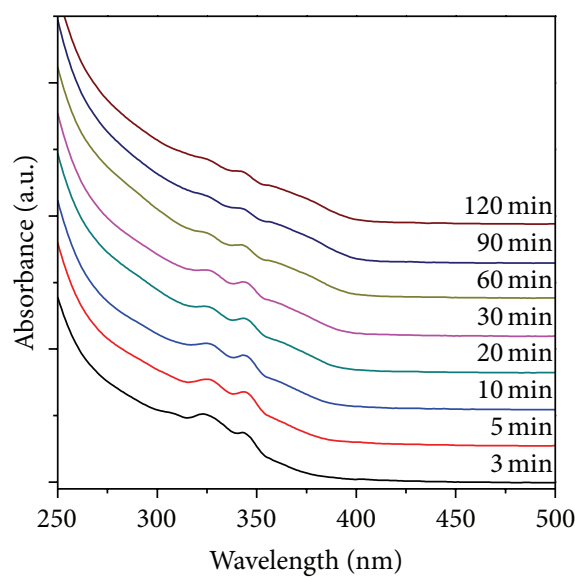

(b)

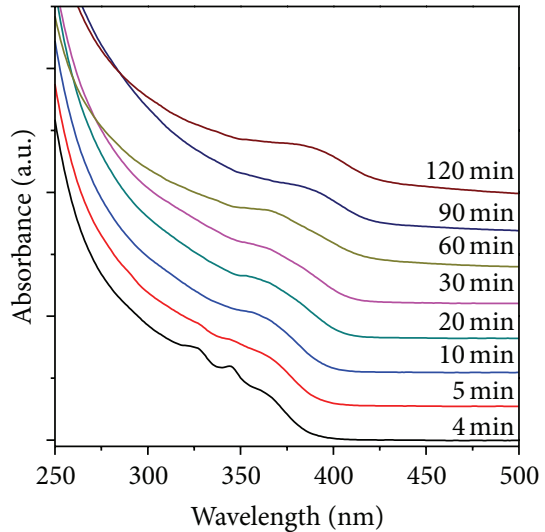

(d)

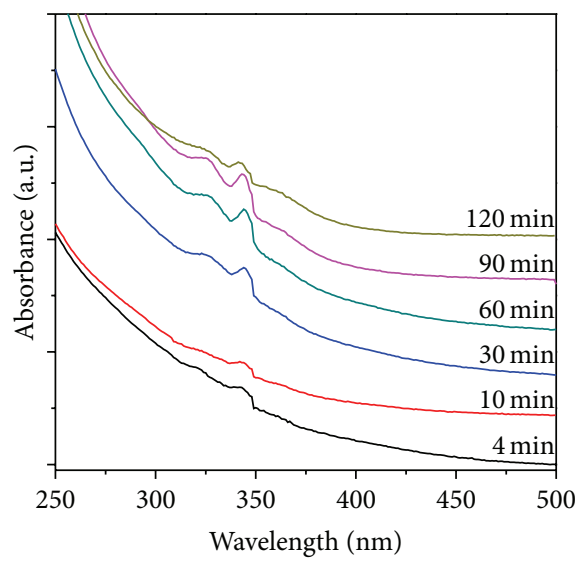

(f)

Figure 1: UV-Vis absorption spectra of ZnSe NCs synthesized at $220^{\circ} \mathrm{C}$ using different Zn precursors: (a) $0.4 \mathrm{mmol} \mathrm{ZnAc}_{2}$, (b) $0.4 \mathrm{mmol}$ $\mathrm{Zn}(\mathrm{NA})_{2}$, (c) $0.4 \mathrm{mmol} \mathrm{ZnSt}_{2}$, (d) $0.6 \mathrm{mmol} \mathrm{ZnAc}_{2}$, (e) $0.6 \mathrm{mmol} \mathrm{Zn(NA)}$, and (f) $0.6 \mathrm{mmol} \mathrm{ZnSt}_{2}$.

the characteristic absorption peaks of $\mathrm{F} 347$ magic-sized $\mathrm{ZnSe}$ NCs synthesized using $0.4 \mathrm{mmol}$ of $\mathrm{ZnAc}_{2}$ within $10 \mathrm{~min}$ can be observed clearly (Figure 1(a)), while the corresponding characteristic peaks only exist in the ZnSe NCs sample collected at $4 \mathrm{~min}$ when using $0.6 \mathrm{mmol}$ of $\mathrm{ZnAc}_{2}$; the excitonic absorption peak position after $60 \mathrm{~min}$ is also shifted from $375 \mathrm{~nm}$ in the $0.4 \mathrm{mmol}$ of $\mathrm{ZnAc}_{2}$ case to $390 \mathrm{~nm}$ in the $0.6 \mathrm{mmol}$ of $\mathrm{ZnAc}_{2}$ case (Figure 1(d)). The same results can be obtained when using $\mathrm{Zn}(\mathrm{NA})_{2}$ and $\mathrm{ZnSt}_{2}$ as $\mathrm{Zn}$ precursors. When using $0.4 \mathrm{mmol}$ of $\mathrm{Zn}(\mathrm{NA})_{2}$, the characteristics peak of F347 magic-sized ZnSe NCs does not disappear completely up to $120 \mathrm{~min}$, although the absorption in the range of $300-400 \mathrm{~nm}$ increases gradually, which means that some F347 magic-sized ZnSe NCs do not transform into regular $\mathrm{ZnSe}$ NCs. However, when adding $0.6 \mathrm{mmol} \mathrm{Zn}(\mathrm{NA})_{2}$, the characteristics peaks of F347 magic-sized NCs can only be 

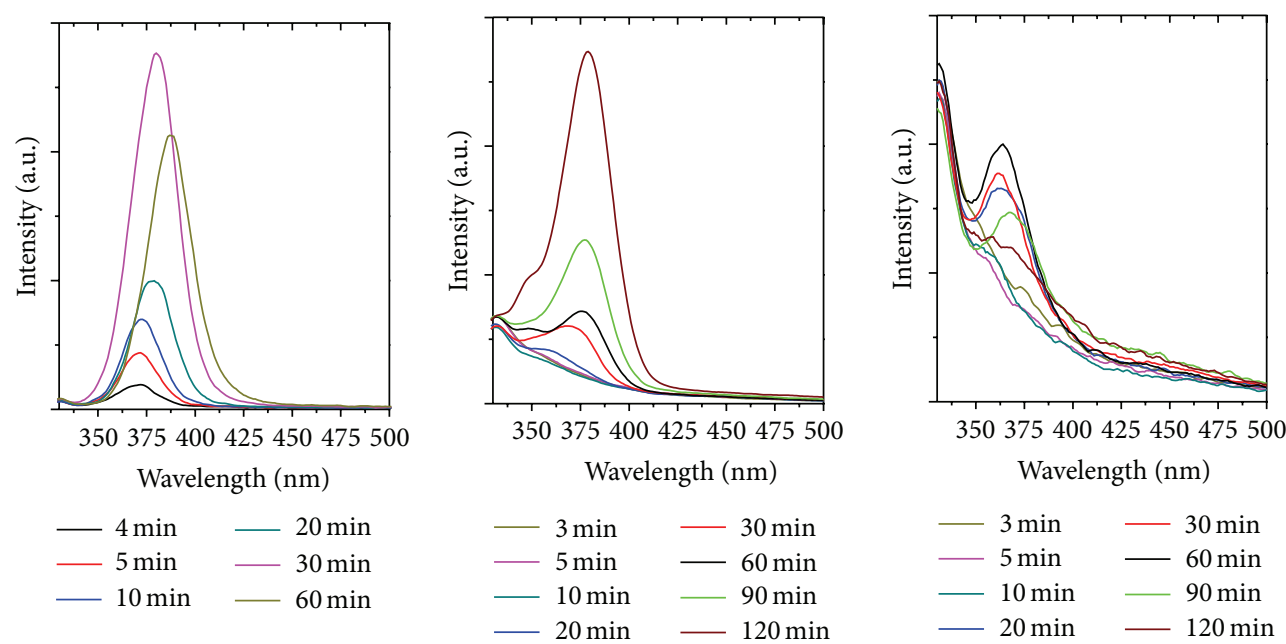

(a)

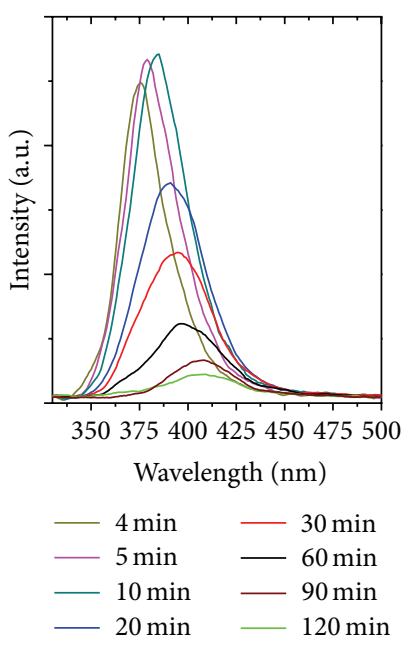

(d)

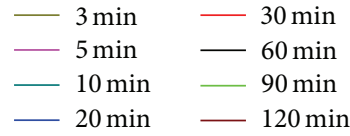

(b)

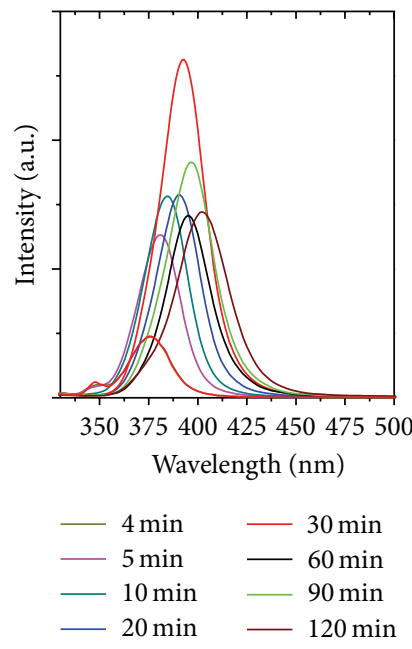

(e) (c)

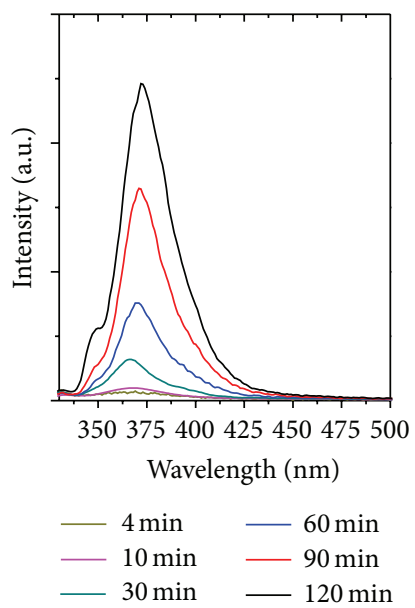

(f)

Figure 2: PL spectra of ZnSe NCs synthesized at $220^{\circ} \mathrm{C}$ using different $\mathrm{Zn}$ precursors: (a) $0.4 \mathrm{mmol}_{\mathrm{ZnAc}}$, (b) $0.4 \mathrm{mmol} \mathrm{Zn}(\mathrm{NA})_{2}$, (c)

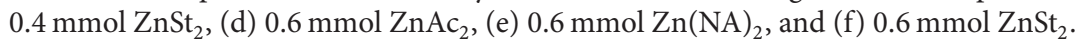

observed within $10 \mathrm{~min}$; then the F347 magic-sized ZnSe NCs will grow up and form the relatively large regular ZnSe NCs with the first excitonic absorption peak above $400 \mathrm{~nm}$ at $120 \mathrm{~min}$. For $\mathrm{ZnSt}_{2}$ precursors, the characteristics peaks of F347 magic-sized ZnSe NCs are always apparent and strong when $0.4 \mathrm{mmol}$ of $\mathrm{ZnSt}_{2}$ is used, while the absorption in the range of $350-400 \mathrm{~nm}$ corresponding to regular ZnSe NCs will become much stronger when using the $0.6 \mathrm{mmol}$ of $\mathrm{ZnSt}_{2}$ as $\mathrm{Zn}$ precursor. All of the above results show that the more the added $\mathrm{Zn}$ precursors, the faster the resulting $\mathrm{ZnSe}$ NCs will grow. The fact that the addition of larger amount of $\mathrm{Zn}$ precursors facilitates faster growth of the resulting ZnSe NCs may be due to very high reactivity of our Se precursor (alkylamine- $\mathrm{H}_{2} \mathrm{Se}$ complex). Thus, controlled and continuous growth of $\mathrm{ZnSe}$ NCs can be achieved not only by using highly reactive and stoichiometric $\mathrm{Zn}$ and Se precursors but also by using larger amount of more unreactive $\mathrm{Zn}$ precursor when the highly reactive alkylamine- $\mathrm{H}_{2} \mathrm{Se}$ complex precursor was used as Se precursor.
3.2. PL Spectrum. PL spectrum is also an important tool for characterizing the optical performance of luminescent semiconductor NCs, which is helpful for obtaining the best nucleation and growth conditions of high-quality ZnSe NCs [14]. Figure 2 shows the evolution of PL spectra of $\mathrm{ZnSe} N C s$ synthesized at $220^{\circ} \mathrm{C}$ using $\mathrm{ZnAc}_{2}, \mathrm{Zn}(\mathrm{NA})_{2}$, and $\mathrm{ZnSt}_{2}$ as $\mathrm{Zn}$ precursors, respectively. By comparing Figure 1 with Figure 2, it can be found that these resulting ZnSe NCs emit bandedge fluorescence other than surface-defect fluorescence, and their corresponding full widths at half maximum (FWHM) are relatively narrow (usually about $25-30 \mathrm{~nm}$ ). As shown in Figure 2(a), when $0.4 \mathrm{mmol}$ of $\mathrm{ZnAc}_{2}$ is used as $\mathrm{Zn}$ precursor, the resulting high-quality ZnSe NCs obtained at the shortest reaction time $(4 \mathrm{~min})$ emit the relatively strong band-edge fluorescence with the peak position at $372 \mathrm{~nm}$, and the PL peak will gradually shift to $387 \mathrm{~nm}$ at $60 \mathrm{~min}$. When $0.6 \mathrm{mmol}$ of $\mathrm{ZnAc}_{2}$ is used, the resulting $\mathrm{ZnSe} \mathrm{NCs}$ will also emit the relatively strong band-edge fluorescence at $375 \mathrm{~nm}$ for $4 \mathrm{~min}$, and the corresponding PL peak will shift to $396 \mathrm{~nm}$ for $60 \mathrm{~min}$ 
and to $408 \mathrm{~nm}$ for $120 \mathrm{~min}$ (Figure 2(d)). However, unlike $\mathrm{ZnAc}_{2}$ as $\mathrm{Zn}$ precursor, there is not any obvious emission from ZnSe NCs synthesized using $0.4 \mathrm{mmol}$ of $\mathrm{Zn}(\mathrm{NA})_{2}$ or $\mathrm{ZnSt}_{2}$ as $\mathrm{Zn}$ precursors during $10 \mathrm{~min}$, and their emission at longer reaction time is also weaker than that corresponding to those obtained by using $\mathrm{ZnAc}_{2}$ as $\mathrm{Zn}$ precursor (Figures 2(b) and $2(\mathrm{c})$ ). These remarkably different PL phenomena may be due to their different steric effects when using different $\mathrm{Zn}$ precursors. In the synthesis of $\mathrm{ZnSe} \mathrm{NCs}$ using $\mathrm{ZnAc}_{2}$ precursor, acetate is a kind of carboxylate with very short alkyl chain and can be easily replaced by alkylamine because of the relative small steric effect of $\mathrm{ZnAc}_{2}$. In comparison with $\mathrm{ZnAc}_{2}$, both $\mathrm{Zn}(\mathrm{NA})_{2}$ and $\mathrm{ZnSt}_{2}$ have larger steric effect because of their longer carboxylate ligands. Generally, relative to the carboxylate ligand reported previously, alkylamine is more favorable to obtain excellent fluorescence performance such as high fluorescence efficiency and narrow fluorescence peak for ZnSe NCs [3, 7].

In addition, due to longer carboxylate ligands of $\mathrm{Zn}(\mathrm{NA})_{2}$ and $\mathrm{ZnSt}_{2}$ compared with $\mathrm{ZnAc}_{2}$, the resultant stronger steric effects of $\mathrm{Zn}(\mathrm{NA})_{2}$ and $\mathrm{ZnSt}_{2}$ make them have lower reactivity than that of $\mathrm{ZnAc}_{2}$. Therefore, in the synthesis using $\mathrm{Zn}(\mathrm{NA})_{2}$ and $\mathrm{ZnSt}_{2}$ precursors, Se precursor cannot be depleted completely, and the excess of Se precursor will adsorb at the surface of ZnSe NCs, which also quench fluorescence from $\mathrm{ZnSe} N C s$. This result is further demonstrated by the fluorescence properties of $\mathrm{ZnSe}$ NCs synthesized using larger amount of $\mathrm{Zn}(\mathrm{NA})_{2}$ and $\mathrm{ZnSt}_{2}$ precursors, as shown in Figures 2(e) and 2(f). When the used $\mathrm{Zn}(\mathrm{NA})_{2}$ or $\mathrm{ZnSt}_{2}$ is added from $0.4 \mathrm{mmol}$ to $0.6 \mathrm{mmol}$, the resulting highquality $\mathrm{ZnSe} \mathrm{NCs}$ will always emit relatively strong band-edge fluorescence with small FWHM comparable with that in the case of $\mathrm{ZnAc}_{2}$, indicating excellent optical property that can be also achieved not only by using highly reactive $\mathrm{Zn}$ and Se precursors with stoichiometric ration but also by using larger amount of more unreactive $\mathrm{Zn}$ precursor than the highly reactive alkylamine- $\mathrm{H}_{2} \mathrm{Se}$ complex precursor. In addition, when using larger amount of $\mathrm{Zn}(\mathrm{NA})_{2}$ and $\mathrm{ZnSt}_{2}$ precursors, the main PL peaks will gradually red-shift from $375 \mathrm{~nm}$ to $402 \mathrm{~nm}$ for $\mathrm{Zn}(\mathrm{NA})_{2}$ case and from $361 \mathrm{~nm}$ to $372 \mathrm{~nm}$ for $\mathrm{ZnSt}_{2}$ with the increase of reaction time during $120 \mathrm{~min}$. Thus, for these three kinds of zinc precursors systems, the red-shift degrees of their fluorescence peaks, with the order of $\mathrm{ZnAc}_{2}>$ $\mathrm{Zn}(\mathrm{NA})_{2}>\mathrm{ZnSt}_{2}$, are obviously dependent on the alkyl chain length of their carboxyl groups, which will coincide with these results of UV-Vis absorption spectra shown in Figure 1. In addition, it is noteworthy that some shoulder peaks at about $347 \mathrm{~nm}$ from the emission of F347 magicsized $\mathrm{ZnSe}$ NCs are also observed in the cases of $0.6 \mathrm{mmol}$ of $\mathrm{Zn}(\mathrm{NA})_{2}$ and $\mathrm{ZnSt}_{2}$ precursors, consistent with the facts that a small amount of F347 magic-sized ZnSe NCs remain in these systems (Figures 1(e) and 1(f)).

\section{Conclusions}

Results of UV-Vis absorption and PL spectra studies on the $\mathrm{ZnSe}$ NCs indicate that the growth and optical property of the resulting $\mathrm{ZnSe}$ nanocrystals are strongly dependent on both the different alkyl chain length and the used amount of the zinc carboxylates. Zinc carboxylate of shorter alkyl chain length leads to faster growth of ZnSe nanocrystals than that of longer alkyl chain length, and for the three kinds of zinc precursors including $\mathrm{ZnAc}_{2}, \mathrm{Zn}(\mathrm{NA})_{2}$, and $\mathrm{ZnSt}_{2}$, the order of the reactivity is as follows: $\mathrm{ZnAc}_{2}>$ $\mathrm{Zn}(\mathrm{NA})_{2}>\mathrm{ZnSt}_{2}$, and their corresponding growth rates of the resulting $\mathrm{ZnSe} \mathrm{NCs}$ follow the same order. In addition, more amount of zinc precursor also leads to faster growth of $\mathrm{ZnSe}$ nanocrystals. These results allow us to tune the growth and optical property of ZnSe NCs by combining the different reactivity and the used amount of the zinc precursor when using the highly reactive alkylamine- $\mathrm{H}_{2} \mathrm{Se}$ complex as Se precursor in the phosphine-free synthesis of high-quality ZnSe NCs. The controlled growth and excellent optical property of high-quality ZnSe NCs can be achieved by using reactivity of both $\mathrm{Zn}$ precursor and Se precursor with closness to stoichiometric ratio or by using larger amount of unreactive $\mathrm{Zn}$ precursor relative to the highly reactive alkylamine- $\mathrm{H}_{2}$ Se complex precursor.

\section{Acknowledgments}

The authors thank the financial support of the Scientific Research Foundation of Shangrao Normal University, Jiangxi Provincial Department of Education (no. GJJ13711), and Department of Science and Technology (nos. 20122BAB203002, 20132BAB203003, and 2012zbbe50006).

\section{References}

[1] M. A. Hines and P. Guyot-Sionnest, "Bright UV-blue luminescent colloidal ZnSe nanocrystals," Journal of Physical Chemistry $B$, vol. 102, no. 19, pp. 3655-3657, 1998.

[2] H.-S. Chen, B. Lo, J.-Y. Hwang et al., "Colloidal ZnSe, ZnSe/ZnS, and $\mathrm{ZnSe} / \mathrm{ZnSeS}$ quantum dots synthesized from $\mathrm{ZnO}$," Journal of Physical Chemistry B, vol. 108, no. 44, pp. 17119-17123, 2004.

[3] L. S. Li, N. Pradhan, Y. Wang, and X. Peng, "High quality ZnSe and $\mathrm{ZnS}$ nanocrystals formed by activating zinc carboxylate precursors," Nano Letters, vol. 4, no. 11, pp. 2261-2264, 2004.

[4] Q. Dai, N. Xiao, J. Ning et al., "Synthesis and mechanism of particle- and flower-shaped ZnSe nanocrystals: green chemical approaches toward green nanoproducts," Journal of Physical Chemistry C, vol. 112, no. 20, pp. 7567-7571, 2008.

[5] S. Acharya, D. D. Sarma, N. R. Jana, and N. Pradhan, "An alternate route to high-quality $\mathrm{ZnSe}$ and $\mathrm{Mn}$-doped $\mathrm{ZnSe}$ nanocrystals," Journal of Physical Chemistry Letters, vol. 1, no. 2, pp. 485-488, 2010.

[6] H. B. Shen, H. Z. Wang, X. M. Li et al., "Phosphine-free synthesis of high quality $\mathrm{ZnSe}, \mathrm{ZnSe} / \mathrm{ZnS}$, and $\mathrm{Cu}-, \mathrm{Mn}$-doped ZnSe nanocrystals," Dalton Transactions, no. 47, pp. 1053410540, 2009.

[7] A. B. Panda, G. Glaspell, and M. S. El-Shall, "Microwave synthesis of highly aligned ultra narrow semiconductor rods and wires," Journal of the American Chemical Society, vol. 128, no. 9, pp. 2790-2791, 2006.

[8] A. B. Panda, S. Acharya, S. Efrima, and Y. Golan, "Synthesis, assembly, and optical properties of shape- and phase-controlled ZnSe nanostructures," Langmuir, vol. 23, no. 2, pp. 765-770, 2007. 
[9] A. B. Panda, S. Acharya, and S. Efrima, "Ultranarrow ZnSe nanorods and nanowires: structure, spectroscopy, and one-dimensional properties," Advanced Materials, vol. 17, no. 20, pp. 24712474, 2005.

[10] P. D. Cozzoli, L. Manna, M. L. Curri et al., "Shape and phase control of colloidal ZnSe nanocrystals," Chemistry of Materials, vol. 17, no. 6, pp. 1296-1306, 2005.

[11] L.-J. Zhang, X.-C. Shen, and H. Liang, "A mild phosphine-free synthesis of alkylamine-capped CdSe nanocrystals," Journal of Nanoscience and Nanotechnology, vol. 10, no. 8, pp. 4979-4984, 2010.

[12] L.-J. Zhang, X.-C. Shen, H. Liang, and J.-T. Yao, "Multiple families of magic-sized $\mathrm{ZnSe}$ quantum dots via noninjection onepot and hot-injection synthesis," Journal of Physical Chemistry C, vol. 114, no. 50, pp. 21921-21927, 2010.

[13] G. N. Karanikolos, P. Alexandridis, G. Itskos, A. Petrou, and T. J. Mountziaris, "Synthesis and size control of luminescent $\mathrm{ZnSe}$ nanocrystals by a microemulsion-gas contacting technique," Langmuir, vol. 20, no. 3, pp. 550-553, 2004.

[14] A. B. Panda, S. Acharya, S. Efrima, and Y. Golan, "Synthesis, assembly, and optical properties of shape- and phase-controlled ZnSe nanostructures," Langmuir, vol. 23, no. 2, pp. 765-770, 2007. 

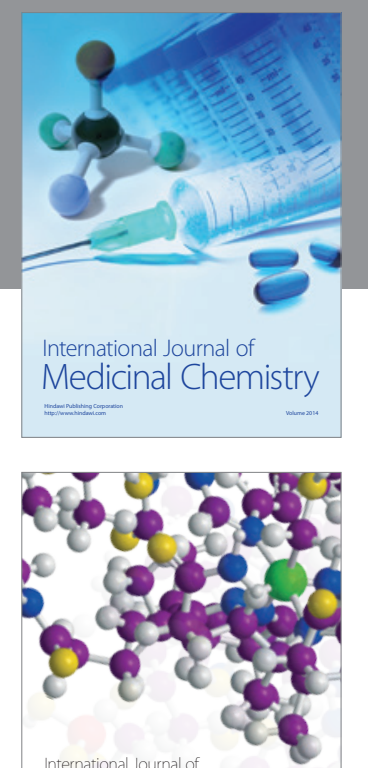

\section{Carbohydrate} Chemistry

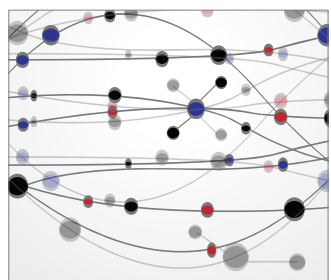

The Scientific World Journal
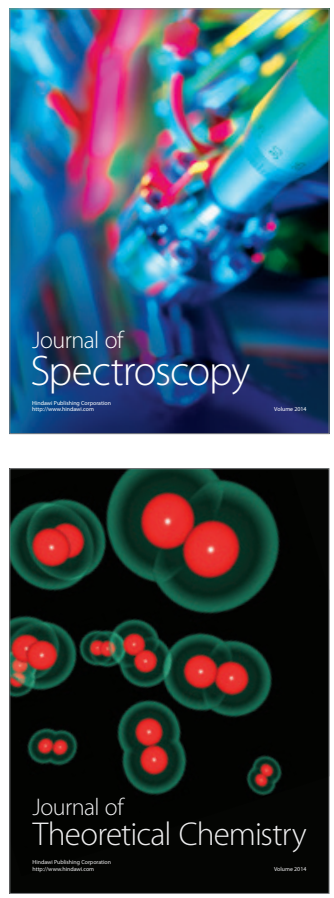
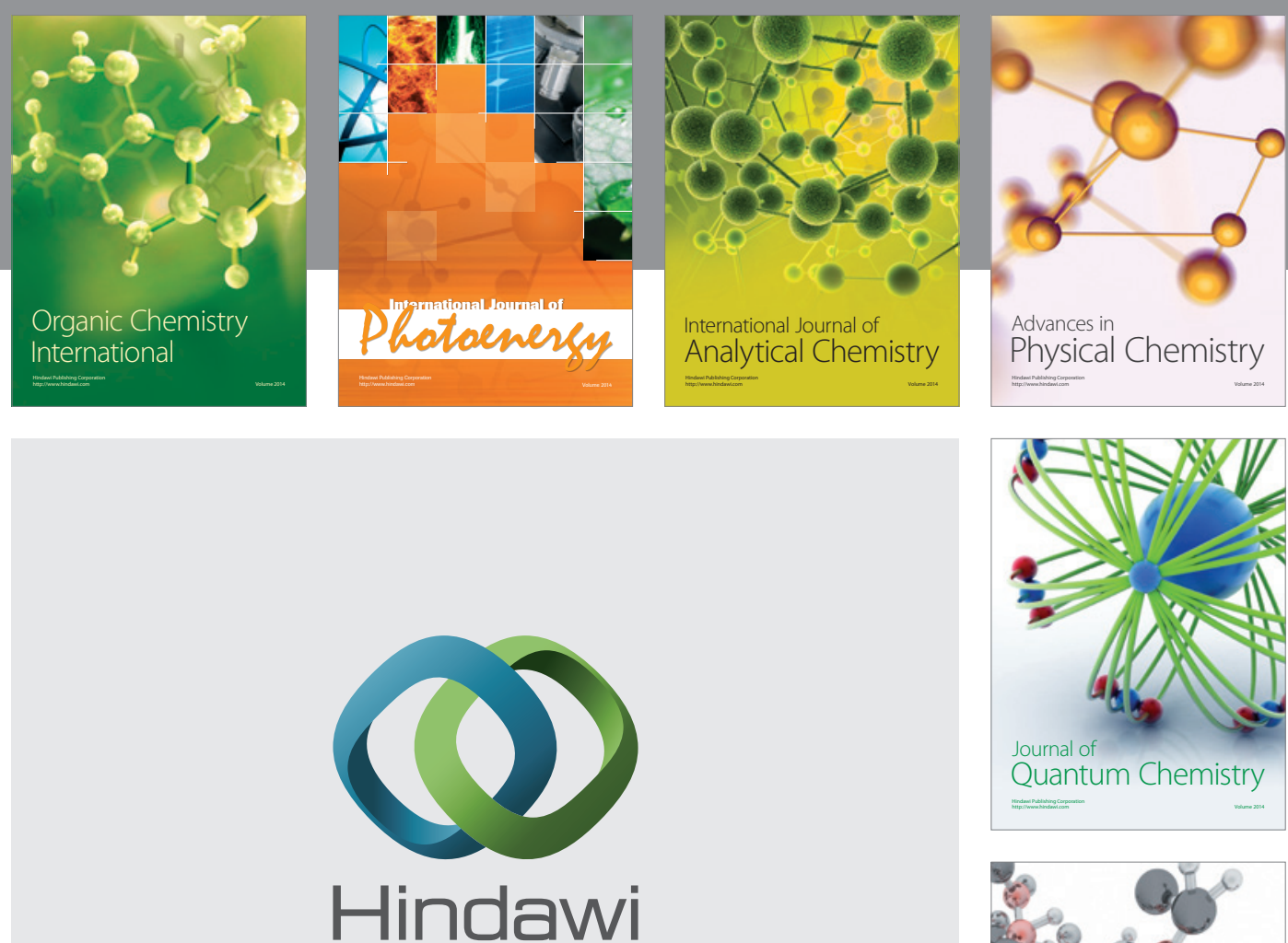

Submit your manuscripts at

http://www.hindawi.com

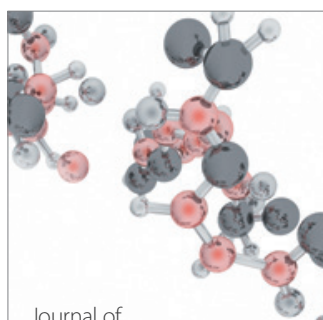

Analytical Methods

in Chemistry

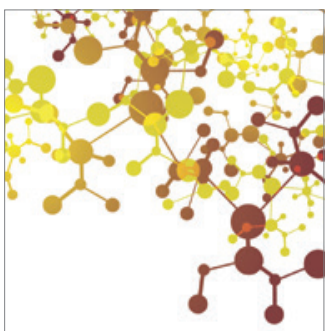

Journal of

Applied Chemistry

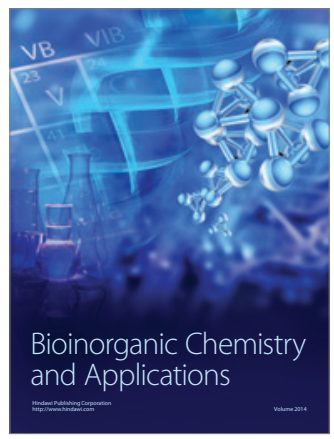

Inorganic Chemistry
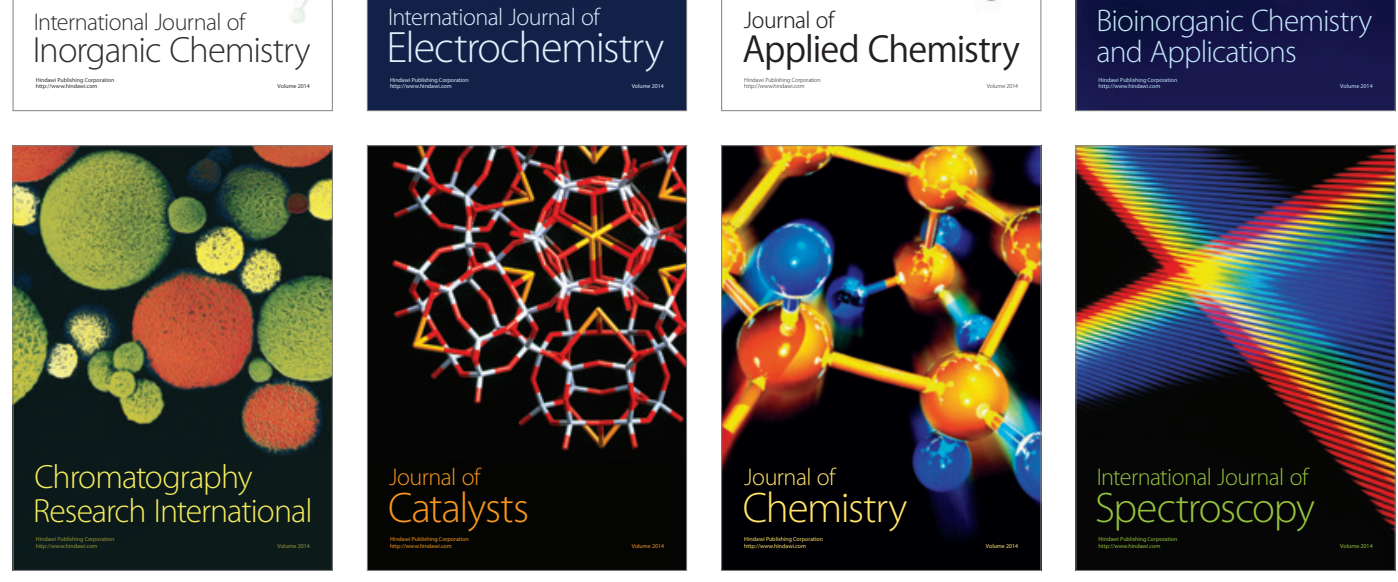